\title{
A probabilistic cognitive simulator for HRA studies (PROCOS)
}

\author{
P. Trucco*, M.C. Leva \\ Politecnico di Milano, Department of Management, Economics and Industrial Engineering, Piazza Leonardo da Vinci 32, Milan 20132, Italy
}

Received 28 March 2005; received in revised form 20 March 2006; accepted 1 June 2006

\begin{abstract}
The paper deals with the development of a simulator for approaching human errors in complex operational frameworks (e.g., plant commissioning). The aim is to integrate the quantification capabilities of the so-called 'first-generation' human reliability assessment (HRA) methods with a cognitive evaluation of the operator. The simulator allows analysing both error prevention and error recovery. It integrates cognitive human error analysis with standard hazard analysis methods (Hazop and event tree) by means of a 'semi static approach'. The comparison between the results obtained through the proposed approach and those of a traditional HRA method such as human error assessment and reduction technique, shows the capability of the simulator to provide coherent and accurate analysis.

(C) 2006 Elsevier Ltd. All rights reserved.
\end{abstract}

Keywords: HRA; Cognitive simulation; Error recovery; Commissioning

\section{Introduction}

"Traditional HRA approaches use a simple classification scheme but have only weak links to a model of cognition. Information processing approaches can produce detail explanations in terms of mental processes, but are weak in accounting for causes that have their origin in the working environment. While cognitive approaches may avoid both problems, they are still under development, in order to be practically applied" [1].

Many efforts have been recently put in the direction suggested by Hollnagel and Marsden [1], trying to integrate the quantification capability of the so-called 'first-generation' human reliability assessment (HRA) methods in safety assessment (i.e., THERP and SLIM) with a cognitive evaluation of the operators involved in the context under examination. As Mosleh and Chang [2] pointed out "compared to the first generation methods, and with respect to the number and scope of applications, the second generation methods are still mostly in development or in trial application stages". Furthermore, they indicate some

\footnotetext{
*Corresponding author. Tel.: + 390223994845 ; fax: + 390223994967

E-mail addresses: paolo.trucco@polimi.it (P. Trucco), chiara.leva@polimi.it (M.C. Leva).
}

of the desirable characteristics of advanced HRA methods such as:

- better explanatory casual models;

- more explicit role for 'context' both in error identification and probability estimation;

- specific and systematic guidelines for identifying errors and recovery actions including the domains as function of the PSFs;

- applicability by different users for different problems;

- traceability, consistency and repeatability.

The present work is an attempt in these directions through a particular use of cognitive simulation.

In the classical probabilistic safety assessment (PSA) framework HRA is still mostly considered through traditional approaches. Traditional HRA approaches are based on detailed task analysis - analysis of every step constituting the task that the operator has to performand a classification of possible human errors. It is possible to construct an event tree with actions to be performed and the possible deviations of the operator. At each branch of the tree a probability value is determined, the value can derive from expert judgments or from data collected in databases, adaptable to the situation of concern. The mental processes followed to choose or to perform a human action are not taken into 
consideration. Thus possible important sources of information are lost.

As Straeter [3] highlights, the following aspects are lacking:

- an appropriate methodological framework which allows to represent relevant error mechanisms as well as contextual and organisational conditions;

- the consideration of the above elements in the quantification approach;

- a sufficient database for the quantification of the error mechanisms as well as for the contextual and organisational conditions.

These kinds of methods can be defined as static because they do not take into account dynamic interactions of the operator with the system. However, traditional HRA approaches have two main advantages:

- they are generalised, i.e., they can be applied to different context and circumstances, partly because of the very absence of a dynamic model of the system, or of the operator, to be analysed;

- They convey quantitative results obtained from a limited amount of effort in terms of processing time and expert personnel.

On the other side information-processing approaches present detailed models for the operator. The operator is seen as an information-processing system for which mental processes are considered as rigorously specifiable procedures and mental states as defined by their casual relation with sensory input, motor behaviour, and other mental states [4]. Furthermore, on the same bank but with a different viewpoint it is possible to mention cognitive approaches. According to Hollnagel [4], the cognitive approach is based on explicit use of models or theories of the cognitive functions which constitute the substratum of human behaviour but, differing from the information-processing approach, cognition is viewed as active rather than reactive and it focused on the overall performance rather than on the mechanism of performance. Therefore, HRA methods in the cognitive approach try to take into consideration the operator, the system and their interactions. In some cases they can be constituted of four different models:

1. a model for task execution, that aims at representing the operator's choice of the actions to be performed;

2. a cognitive model for the operator. This model determines if the action to be executed will be correct or wrong, and in the latter case the possible error modes are not determined 'a priori', they are functions of the parameters utilised in the cognitive model itself;

3. a dynamic model of the system, able to change the main parameters of status of the system, following an operator's action;

4. a model for the operator-context interaction.
Cognitive models can help in analysing human mental processes that can lead to error. They do not bind operator choices to a system evolution already established through an event tree, in this sense they can be referred to as dynamic models. Unfortunately quantitative HRA methods in the cognitive approach, as already stated, are mostly still under trial, they are often tailored on the specific context they refer to and thus their approach is difficult to generalise for applications in different contexts. One of the main applications stemmed from the cognitive approach is the development of the simulations of cognition or cognitive simulations.

\section{State of the art: review of HRA by means of simulation approaches}

A cognitive simulation consists of the reproduction of a model of cognition using a numerical application or computation. While the model of cognition is the theoretical representation of the mental processes and control actions developed by one or more operators during the execution of their tasks, given a physical system and a definite context [5].

A cognitive simulation can be quantitative or qualitative. A qualitative simulation describes the evolution of a cognitive process, i.e., from the reception of an external stimulus to the subsequent action, whereas a quantitative one is based on the structure of a qualitative one with the addition of a computational section. The qualitative study in this case is often coupled with a simulation of the performance of the system the operator has to interact with. The final outcome of a quantitative simulation can be the list of the types of actions or errors performed by the operator, while executing a specific task, or a probability value for each type of action, calculated through the simulation runs. Cognitive simulators developed up to now have been mainly used for qualitative analysis and they have not found large applications in the quantitative risk assessment framework. On the other hand, those able to provide quantitative results are tailored on very specific applications and coupled with simulations of the plant the operator performance should be referred to, therefore very difficult to use for different systems or scenarios. A detailed description of all the cognitive simulations already developed in literature is beyond the aim of the present paper; however a list of the main simulation projects are listed in Table 1, and some of their main features are reported in Table 2.

Not all the cognitive simulators reviewed obtain quantitative results, all of them have been developed for a specific context (e.g., aviation or nuclear power plants) and application (e.g., single operator or team simulation). They are all dynamic, thus they do not bind operator choices to a system evolution already established. Sometimes the models are not easy to understand, and therefore used, by HRA specialists that have not been directly involved in their development. The specificity of the models 
Table 1

Review of main cognitive simulators

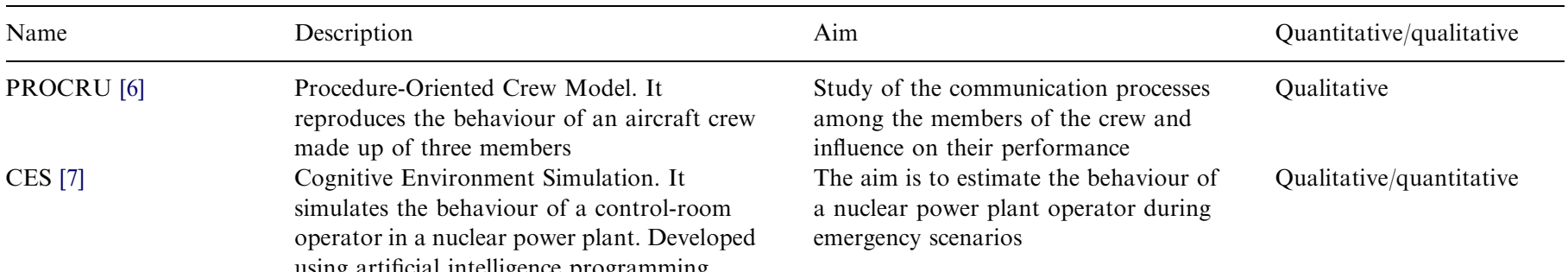

COSIMO [8] Cognitive Simulation Model. It simulates the behaviour of an operator reproduced through the Fallible Machinemodel by Reason [9], coupled with a model for the system specific for the system to be considered

MIDAS [11] Man Machine Integration Design and Analysis system. It can simulate the behaviour of a pilot for civil aviation or an air traffic controller. The model of the operator is based on Rasmussen's model [10]

SYBORG [12] Simulation System for Behaviour of an Operating group. It simulates a group of nuclear power plant operators

Study the operator actions in abnormal

Qualitative/quantitative plant conditions (accident scenarios) in a nuclear power plant

TOPAZ [14]

Scenario and Monte Carlo simulation-based accident risk assessment of an ATM operation. The actual safety assessment starts by determining the operation that is assessed. Next, hazards associated with the operation are identified, and clustered into conflict scenario. Using severity and frequency assessments and the risk associated with each conflict scenario is classified

TBNM [15]

Team Behaviour Network Model. The simulation is made up of three components: a model for the task to be executed, the model of the event development after an initiating event and the model of the team which comprise a human machine interaction model as well

AITRAM [13] Advanced Integrated Training in Aeronautics Maintenance) As part of the European Project an AITRAM simulator has been introduced able to provide information regarding the possible errors that an aircraft maintenance technician can commit

The aim of the simulator is to study the interaction between the operator (pilot or air traffic controller) and the external environment

It highlights some possible combinations of operator errors and plant condition that can lead to accident sequences; it proposes different strategies to improve the collaboration within the group

For each conflict scenario with a possibly unacceptable risk, safety bottlenecks are identified. It aims at enabling context-related human reliability analysis at cognitive level, and it is combined with an aircraft collision risk models

The core of the simulation is to study the cognitive process of the team which consists in recognising the symptoms, the related decision making and the subsequent planning and execution of the action

It enables to identify the most critical tasks for an operator, thus the results can be used to decide which scenario is worth to reproduce in virtual reality for the technician training

Quantitative

Qualitative

Quantitative

Qualitative

Qualitative

modify only the state of some equipment of the plant according to:

1. a limited set of the states in which the equipment can be turned;

2. the error modes identified through the Hazop and extracted as a result of the cognitive simulation of the operator;

3. an explicit relation between the actions outcomes (correct execution or error modes) and equipment status modifications (the relations are derived from the Hazop analysis). 
Review of main Cognitive Simulators according to a set of criteria

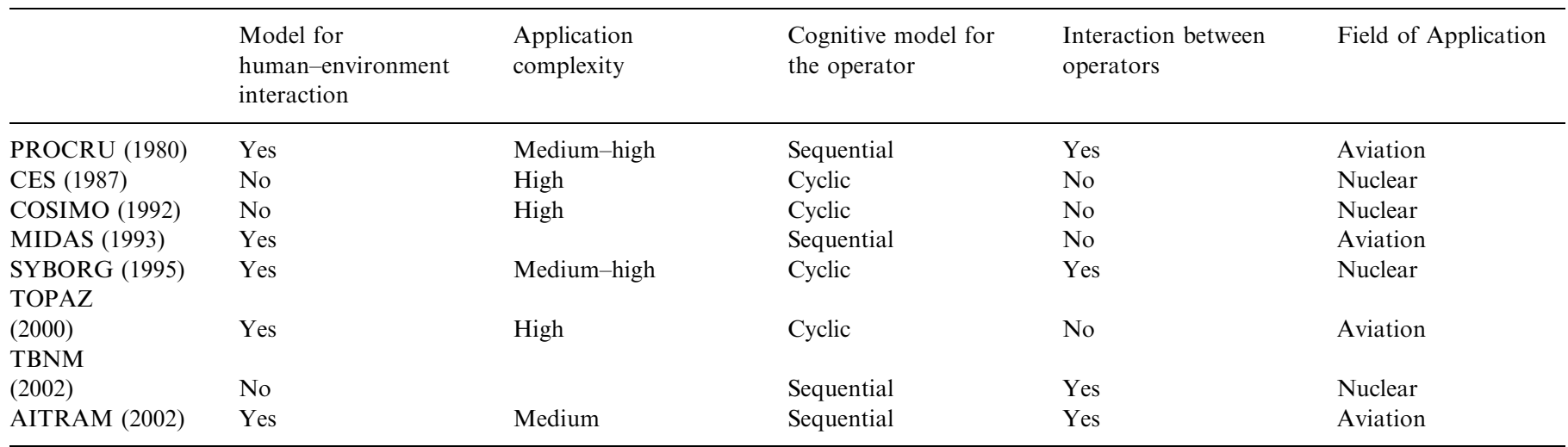

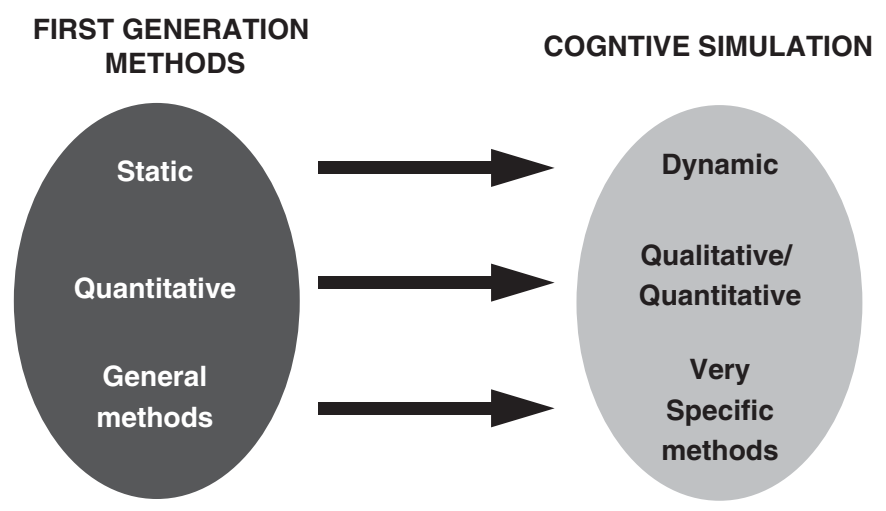

Fig. 1. A comparison between first-generation HRA approaches and cognitive simulation approaches.

FIRST GENERATION METHODS

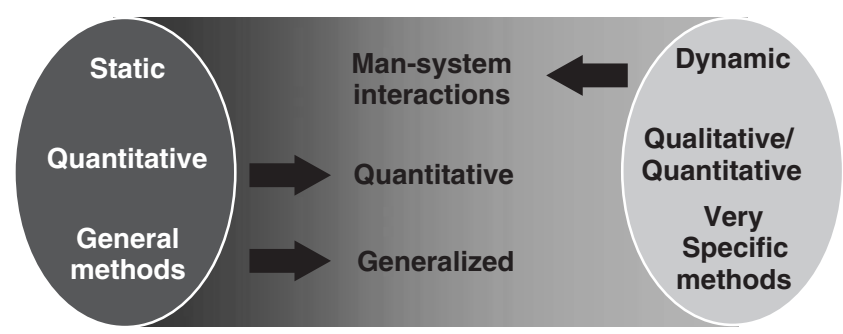

Fig. 2. Characteristics of the semi-static approach.

Its focus is mainly in conveying a quantitative result, comparable with those of a traditional HRA method, taking into account a cognitive analysis of the operator as well. As a further step the simulator considers the evaluation of error management as part of the overall assessment from the same cognitive point of view, differing from the way traditional human reliability methods (e.g., THERP) consider the recovery phase.

PROCOS does not imply the development of a detailed model for the operator-context interaction; the context is taken into account mainly through the input coming from the PSA framework it belongs to, and through the use of performance shaping factors (PSFs), as proposed in traditional HRA methods. However, using the simulation process in PROCOS a mathematical model is proposed for studying how the PSFs influence the operator cognitive process of actions and, therefore, human error probability (HEP). This can trace the way of a cost-benefit analysis for possible corrective actions concerning the entire organisation. The results presented in this paper, refer to the case study of the commissioning phase for an ammonia urate plant, nevertheless, the simulator can be used for analysing different contexts as well.

The simulator has been developed having as a main term of comparison the work done in the EU Joint Research Centre (JRC) at Ispra, Italy [13] during the Advanced Training systems for Aeronautical Maintenance Technicians (AITRAM) Project [16]. However, the simulator proposed differs from the AITRAM one because it introduces:

- different flow charts to simulate the commissioning operator behaviour in normal operations;

- a new flow chart for the recovery phase;

- a different mathematical model for the decision blocks criteria.

The simulator required to develop the following elements:

- a preventive risk analysis of the activity in which the operator action needs to be considered. In the case of study the analysis has been carried out through the Multilevel Hazop [17] methodology and a related event tree (Fig. 3);

- a cognitive model of the operator based on SHEL [18] and PIPE [5];

- a taxonomy of the possible error type in the operation phase (exits of the cognitive flow chart) and the construction of a matrix incorporating the relations between the cognitive error type and the manifestation of the error through the error mode considered in 


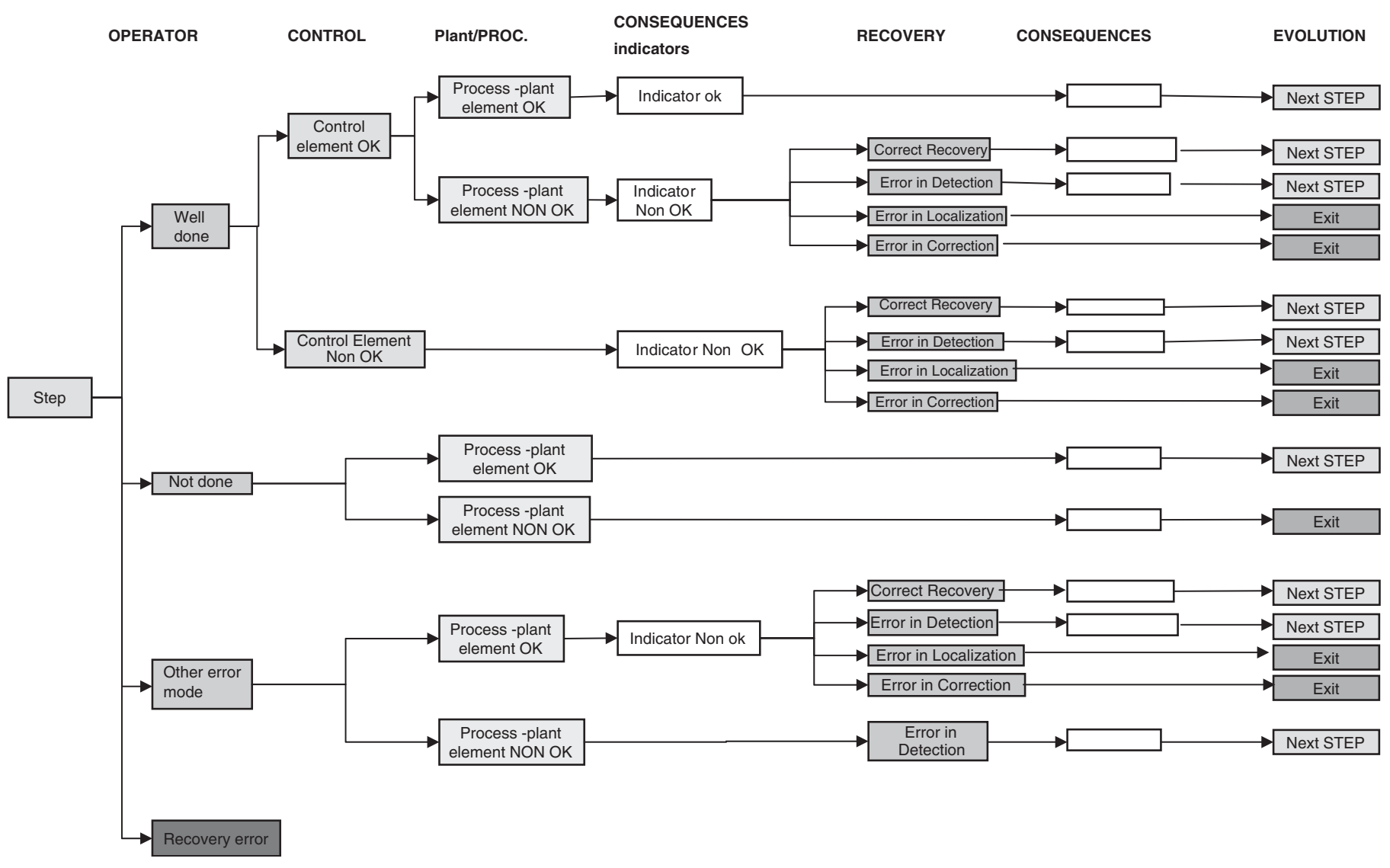

Fig. 3. Event tree for step C.3.13 b of the commissioning procedure.

Human Hazop analysis (guide words); a taxonomy for the possible error type outlined in the recovery phase;

- a cognitive model for the evaluation of human behaviour in the recovery phase based on the framework proposed by Kontogiannis [19];

- The architecture of the cognitive simulator: flow charts to simulate the behaviour of the operator (in normal operations and in the recovery phase) and a mathematical model for the decision block criteria.

\section{Cognitive model of the operator}

Following the definition of 'Minimal Modelling Manifesto' given by Hollnagel [4], which is a representation of the main principles of control and regulation that are established for a domain- as well as for the capabilities and limitations of the controlling system, the method tried to choose a model able to take into account only those aspects that can be considered relevant in order to analyse the operator behaviour and the man-machine interaction. The SHELL model, introduced by Edwards [18] and then developed by Hawkins [20], describes the interaction between procedures (software), equipment (hardware), environment and plants present in the working environment, and the operator (liveware), taking into account the

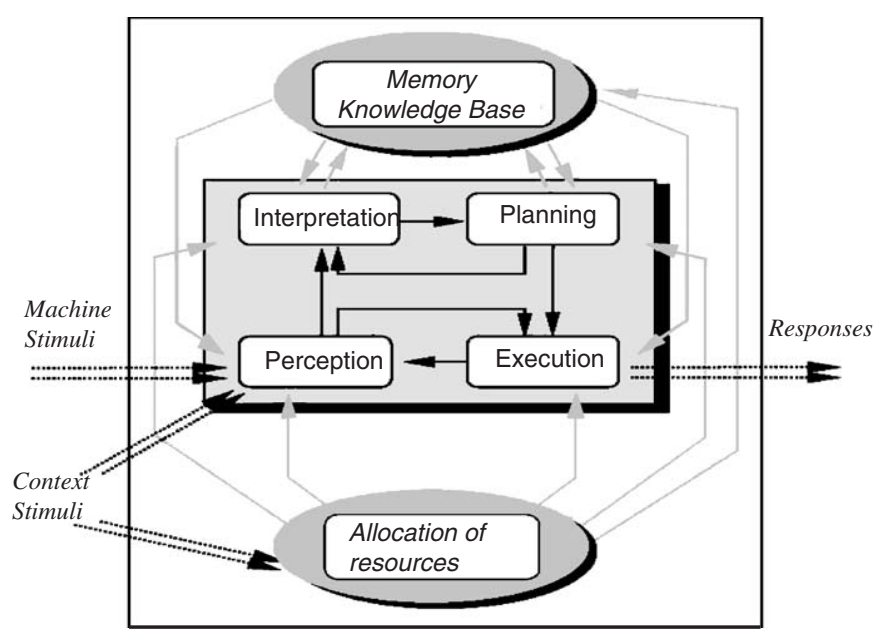

Fig. 4. PIPE model [5].

possibility of interaction of the operator with other operators or supervisors (liveware). In the proposed model all these elements are mainly developed through the links between the elements themselves and the performance shaping factors PSF, influencing the operator's action.

Furthermore the PIPE model (Fig. 4), developed by Cacciabue [5], has been used as a reference for a more detailed configuration of the operator. For the sake of brevity, the model, which is based on the four main 
cognitive functions - perception, interpretation, planning and execution - will not be described in detail in the paper. The cognitive functions are influenced by input parameters such as hardware stimuli and context stimuli. The human cognitive path followed, passing through these cognitive functions, leads to a response (output). The two cognitive processes involved are memory/knowledge base and allocation of resources [5].

The two models have been combined as already proposed in the AITRAM project, since during the execution of the task the operator interact with hardware, software, environment and other operators through his cognitive functions, which are, on the other side, influenced by the stimuli coming from the above elements. The output or 'response' given by a cognitive model are correct actions and error types defined on the basis of the cognitive process that leads to their occurrence and not on their manifestation.

On the other hand, the error mode that we need to link as possible response of the cognitive model used are those previously outlined during the assessment performed with the Multilevel Hazop (Table 3).

The error types chosen as possible output of the cognitive process have been taken by Wickens [21] and consist of:

Error in perception: errors regarding issues related to the picking up and understanding of information;

Error in memory: errors related to both short-term storage and more permanent information based on the person's training and experience;

Error in decision: errors related to the judgement and decision-making process required to the operators;

Error in response: it is sometimes possible to carry out actions that have not been intended, an example of this is often referred as a slip of the tongue.

Table 3

Error modes presented in the Hazop analysis for the operator level

\begin{tabular}{ll}
\hline Error mode & Description \\
\hline Not done & $\begin{array}{l}\text { The action is not performed or the operator has } \\
\text { been unable to perform it } \\
\text { An activity or part of an activity is executed in the } \\
\text { wrong order }\end{array}$ \\
Other than & $\begin{array}{l}\text { The wrong activity is performed } \\
\text { Quantitative: the result of an activity is below the } \\
\text { required level }\end{array}$ \\
More than & $\begin{array}{l}\text { Quantitative: the result of the activity is above the } \\
\text { required level }\end{array}$ \\
Sooner than & $\begin{array}{l}\text { An activity is performed faster than it should have } \\
\text { been in the related scheduling }\end{array}$ \\
An ater than & $\begin{array}{l}\text { An activity is performed slower than it should have } \\
\text { been in the related scheduling }\end{array}$ \\
Part of & $\begin{array}{l}\text { Part of the activity has been omitted } \\
\text { The same activity is repeated a second time } \\
\text { Repeated }\end{array}$ \\
Opposite & $\begin{array}{l}\text { An activity is performed on the opposite way } \\
\text { the expected one }\end{array}$ \\
As well as &
\end{tabular}

Table 4

Correlation level between error modes and cognitive error types

\begin{tabular}{lllll}
\hline Error mode & Error type & & \\
\cline { 2 - 5 } & Perception & Memory & Decision & Response \\
\hline Not done & Medium & Medium & Strong & \\
Misordered & & Strong & Strong & Medium \\
Other than & Medium & Medium & Medium & Medium \\
Less than & Strong & Weak & & Medium \\
More than & Strong & Weak & & Medium \\
Faster & Weak & & & Medium \\
Slower & Weak & & & Medium \\
Sooner than & & & Medium & \\
Later than & Medium & & Strong \\
Repeated & & Strong & & Medium \\
Opposite & & Medium & Medium & \\
As well as & & Medium & & \\
\hline
\end{tabular}

The error types have been linked with the error modes of the Human Hazop through a correlation matrix (Table 4), and for each error type one of the available error modes is extracted for every operator action in a simulation run.

As far as the recovery phase is concerned the taxonomy proposed by Kontogiannis [19] has been chosen, breaking down the error-handling process in three phases: detection, localisation or explanation and correction.

Error in detection: the error happens in the phase in which the error is detected. The detection can take place at different stages of the task execution:

- detection in outcome stage;

- detection in execution stage;

- detection in planning stage.

Error in localisation or explanation: the error takes place in the phase in which after having detected the error, the operator tries to identify its causes.

Error in correction: the error occurs in the phase in which the operator, after having considered the situation, develops and executes an action plan in order to recover from the error. There is no need to relate these error types with Human Hazop error modes since the safety assessment for any possible recovery stage has not been developed with the Multilevel Hazop in the same way as for the normal operation phase. It will be carried out mainly through the use of the simulator.

\section{PROCOS: architecture and computational model of the simulator}

A new operator simulator has been developed introducing two different flow charts: one reproduces the commissioning operator's behaviour in normal operational phase and one in recovery phase. The flow charts are based on the model of cognition previously illustrated (SHELL-PIPE), and a connection module has been added for putting in relation the exit of the cognitive flowchart 
(error type) with the elements to be quantified in the event tree (error modes). The recovery flow chart differs from the normal operation flowcharts in its exits and does not require any additional module. It is linked to the first one through the use of a decision block regarding a possible equipment fault state recognisable at the beginning or at the end of each subtask execution. The structure of the simulator (Fig. 5) is based on:

- the operator module, which implies the cognitive flow charts for Action execution and recovery phase, plus the error types/error modes matrix. The critical underlying feature of this module is the mathematical model for decision block criteria of the flow charts;

- the task execution module, based on the event tree referred to the procedure that has to be simulated;

- the human-machine interface module, made up of tables regarding the hardware state and its connection with the operator actions (task executed or error modes committed).

The inputs required for the simulation process are:

- PSFs affecting the task to be simulated;

- hardware involved in the execution of the task and its possible states;
- steps of the task (task analysis);

- set of error modes to be considered.

The input regarding the hardware involved in task execution simply means the equipments to be used within the task to be simulated at every step, therefore the analyst is required to insert the hardware involved and its possible states, the probability of the hardware to be in a failure state independently from human actions and if the failure is recoverable, the state expected before starting the step of the task for the hardware and the state expected at the end of the correct step or at the end of the failure modes available for the step under analysis. This information is supposed to be available for the analysts. The simulator, in fact, is meant to be used within a PSA context, where the value for possible hardware failures can be taken as an input. The possible path failure in the human-machine interaction that need to be analysed have been already highlighted within the PSA context. The simulation process then provides a probability value in respect of operator actions for every path of the event tree (with multiple trial generation) and a probability value for the corrective action in the recovery phase as well. These probability values depend on the PSFs, directly connected to the decision boxes of the flow charts. In this way it is possible to take into account a cognitive point of view in the HEP

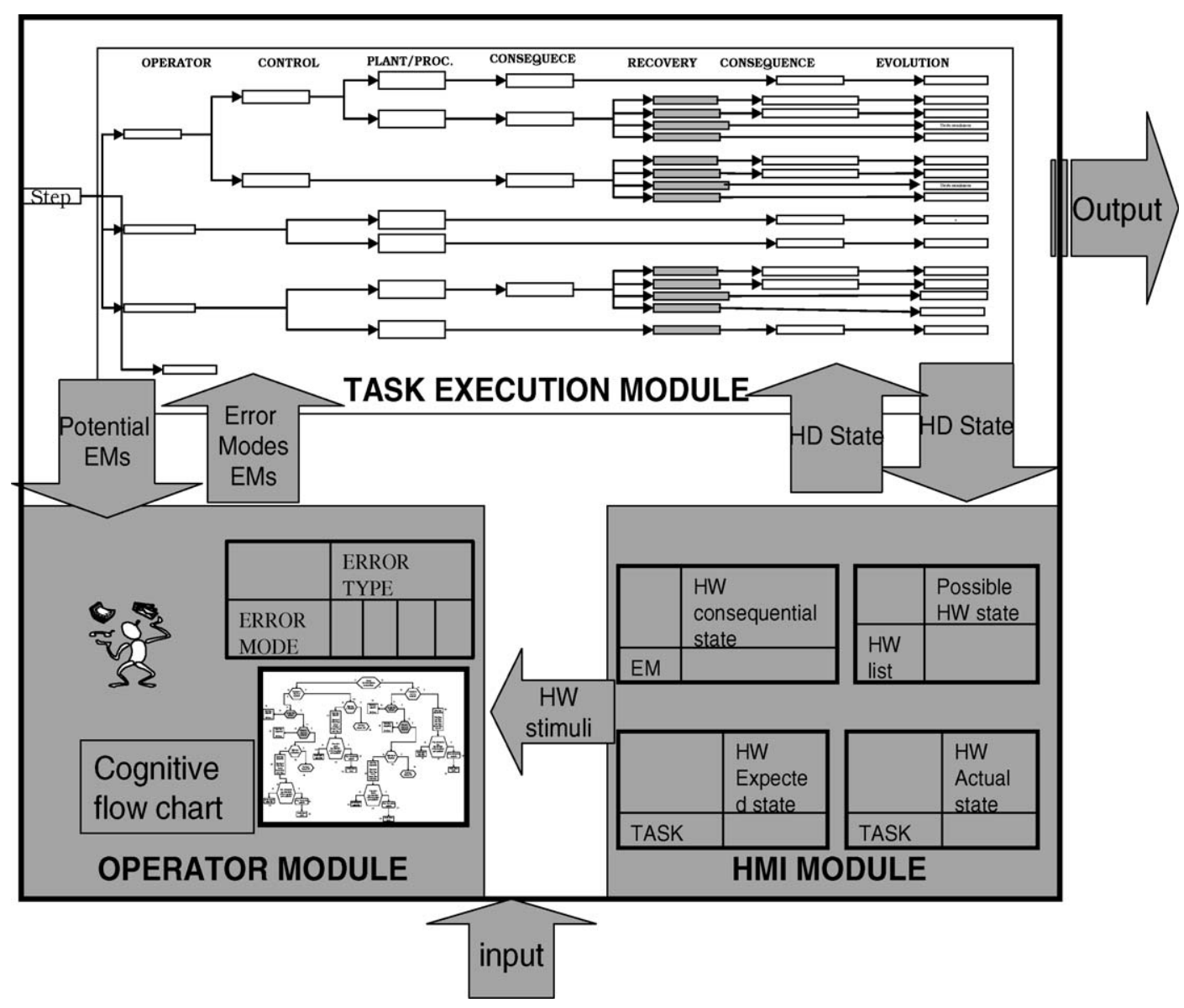

Fig. 5. Structure of the simulator. 
Table 5

List of the performance-shaping factors (PSFs) considered in the analysis

Environmental PSFs

Operator PSFs
Operation planning

Roles and responsibility distribution

Supervision and additional assistance

Workplace conditions

HMI adequacy

Availability of plan and procedures

Number of simultaneous goals

Time available

Fatigue

State of anxiety

Training/experience

Tendency for risky behaviour

Communication/collaboration generation, enabling to consider a more formalised connection with the PSFs, which are the key points for identifying organisational corrective actions. The PSFs considered in the analysis are illustrated in Table 5.

The evolution of the simulation process, as a whole, is managed through a single flow chart, which incorporates all the modules described earlier. The main component of the single flow chart used for the execution of the simulation process is the action flow chart. The action flow chart is a decision blocks diagram, through which it is possible to represent the succession of cognitive functions used by the operator in order to execute an action. According to the path followed in the action flow chart it is possible to identify an error type that depends on the course taken through the decision blocks. Each simulation process runs one action execution flow chart for each step of the procedure to be simulated.

\subsection{Decision blocks criteria}

Each decision block has two possible exits: 'Yes' and 'No'. The exit process is stochastic, described by a Bernoulli's distribution (Yes $\rightarrow x=1$; No $\rightarrow x=0$ ), where the parameter $p$ depends on the PIFs values and the influence they have on each decision block. Then the probability density function $f_{x}(x)$ is equal to

$f_{x}(X)=f_{x}(x, p)= \begin{cases}p^{x}(1-p)^{1-x} & \text { per } x=0 \text { or } x=1, \\ 0 & \text { otherwise }\end{cases}$

where $0 \leqslant p \leqslant 1$ and $q=1-p$.

The probability of having 'Yes' as a possible exit of the block can be expressed as $[P(X=1)]$ and it is equal to $p$, while the probability of having the 'No' exit is $[P(X=0)]$ equal to $q$.

In order to calibrate each decision block, the value of $p$, the success probability of the cognitive process in the block, has been expressed as a function of the PIFs involved for the block (thus also in order to evaluate the influence for the context on the cognitive process).

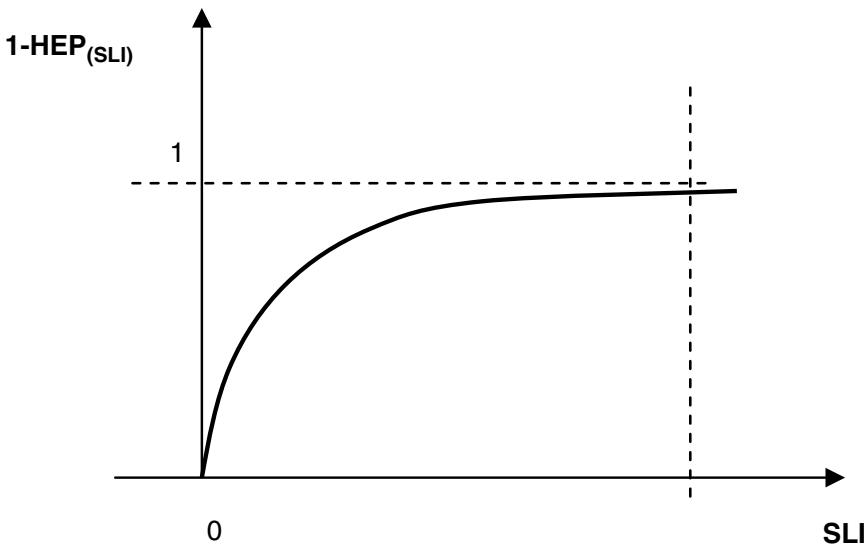

Fig. 6. Mathematical relation between HEP and SLI.

The SLIM method [22] has then been chosen, in particular the expression that relates HEP with a success likelihood index (Fig. 6), which is a logarithmic function of the PSFs involved (formula 2), since in this approach it is accepted that changes in human responses induced by changes in external conditions can be described by a logarithmic relationship [23]

$\log _{10}(\mathrm{HEP})=a \mathrm{SLI}+b$,

where HEP is the human error probability, SLI is the success likelihood index, and $a$ and $b$ are the parameters of the function.

The SLI index is defined as follows:

$\mathrm{SLI}=\sum_{i=1}^{N_{j}}\left(w_{i j} r_{i}\right)$

under the condition

$\sum_{i=1}^{N_{j}}\left(w_{i j} r_{i}\right)=1$

where $w_{i j}$ is the normalised weight of the $i$ th PSF for the cognitive process of the $j$ th block, $r_{i}$ is the $i$ th PSF value, and $N_{j}$ is the number of PSFs considered for the $j$ th block.

For each decision block the HEP value has been taken from the THERP data tables [24], chosen for an error type representative of the cognitive aspect described in each decision block. The value of the median has been used in order to calculate the two parameters $a$ and $b$, from the formula (2), in correspondence to a SLI mean (SLI mean) value for the nominal working condition (central value of the interval for each PSF involved). The second condition was to consider $\mathrm{SP}=0$ for $\mathrm{SLI}=0$ as a bound condition, obtaining $b=0$ (Table 6).

In this way it is possible to determine the probability of each exits from the blocks using the SLI index:

$q=1-p=1-\mathrm{SP}_{\text {block }}$,

$1-\mathrm{SP}_{\text {block }}=\mathrm{HEP}$, 
Table 6

Values of parameter $a$ for each decision block

\begin{tabular}{llll}
\hline Block number & Block type & SLI $_{\text {MEAN }}$ & 1-SP \\
\hline 1 & Hardware/liveware & 55.9 & 0.001 \\
& perception? & & 0.001 \\
3 & Analyse_system? & 62.9 & 0.003 \\
4 & Recognise_stimuli? & 55.65 & 0.003 \\
6 & Correct_H/L_interpretation? & 58.5 & 0.03 \\
8 & Remember_step? & 59.05 & 0.01 \\
9 & Analyse_system_M? & 53.3 & 0.003 \\
11 & Planned_step? & 57.85 & 0.025 \\
20 & Right_step_intention? & 59.2 & 0.003 \\
23 & Correct_perception? & 53 & 0.003 \\
43 & Correct_response? & 64.84 & 0.004 \\
1001 & Hardware/liveware & 59.6 & $-4.536 \mathrm{E}-02$ \\
& perception_R? & & $-2.512 \mathrm{E}-02$ \\
1004 & Analyse_system_R? & 61.7 & $-3.752 \mathrm{E}-02$ \\
1005 & Correct_interpretation_R? & 61.5 & $-4.361 \mathrm{E}-02$ \\
1007 & Localisation? & 59.55 & $-2.706 \mathrm{E}-02$ \\
1008 & Right_localisation? & 59.55 & $-4.760 \mathrm{E}-02$ \\
1009 & Correction_planned? & 61.2 & $-3.891 \mathrm{E}-02$ \\
1011 & Right_correction_planned? & 60.55 & $-4.023 \mathrm{E}-02$ \\
& & & 0.009 \\
\end{tabular}

where $\mathrm{SP}_{\text {block }}$ is the success probability of the block under analysis and HEP is taken from the formula (2).

At the beginning of a simulation process, the values of $\operatorname{PSF}\left(r_{i}\right)$ are extracted as a random variable from a uniform distribution in an interval $R_{i, \min }-R_{i, \max }$, where $R_{i, \min }$ and $R_{i, \max }$ are established according to experts' judgment in connection with the scenario the simulation refers to. In the same way the weights $w_{i}$ are extracted from corresponding estimated intervals $W_{i, \text { inf }}-W_{i, \text { sup }}$.

The simulation study that has been performed for the case under analysis has been run for three main scenarios. For each scenario the PSFs values have been set accordingly through commissioning personnel judgments. The three scenarios analysed are:

(1) optimal case scenario;

(2) nominal conditions scenario;

(3) worst case scenario.

Each simulation campaign consisted of seven simulations each one composed by 10.000 simulation runs.

From the Central Limit Theorem the parameter $p$ of the Bernoullian distribution in formula (1) can be calculated as the mean of the $x$ variable distributed according to a normal distribution. The confidence interval of $p$ at $90 \%$ is illustrated in Table 9.

\section{Case study: HR simulation of a commissioning procedure}

The case study refers to the reliability analysis of the control-room operator of an ammonia urate plant during the commissioning phase, which begins after construction and concerns both machinery tests and plant start-up. It is one of the most critical phases of the life cycle of a project: it demands greater specific knowledge, decision making
Table 7

Commissioning operations under analysis

\begin{tabular}{ll}
\hline Operation & Duration (h) \\
\hline $\begin{array}{l}\text { Warming up of the high pressure section (hot bolting } \\
\text { included) }\end{array}$ & $10-12$ \\
$\begin{array}{l}\text { Draining of the condenser before pressurising. } \\
\text { Pressurising with } \mathrm{NH}_{3} \text { through the start-up line }\end{array}$ & $\frac{1}{2}$ \\
(ammoniation) & $3 \frac{1}{2}$ \\
$\mathrm{NH}_{3}$ and $\mathrm{CO}_{2}$ introduction in the reactor until the & $2-3$ \\
overflow condition is reached & $2-3$ \\
Transition to a stable condition &
\end{tabular}

and managerial skills. Operator errors during execution of plant-commissioning procedures have evident negative consequences such as delay in completion, waste of process materials, physical damage to the plant, inability to operate (at least for a certain period of time) under standard conditions, environmental pollution, serious injury to operators, damage to company image and contract penalties [17].

The present work is placed within the risk analysis realised on the chemical process plant of concern. Its commissioning procedures have been analysed and they have been broken down into single steps during a Multilevel Hazop analysis, that has pointed out the most critical parts of the overall commissioning procedure. Two specific procedures that refer to the start-up operations (Table 7) have been chosen in order to carry out the simulation.

In particular the simulation process has been focused on the steps regarding the introduction of $\mathrm{NH}_{3}$ in the reactor, which interests the part of the plant illustrated in Fig. 7:

Step 1: open the manually operated valve on the adduction pipe to the $\mathrm{NH}_{3}$ pump; 


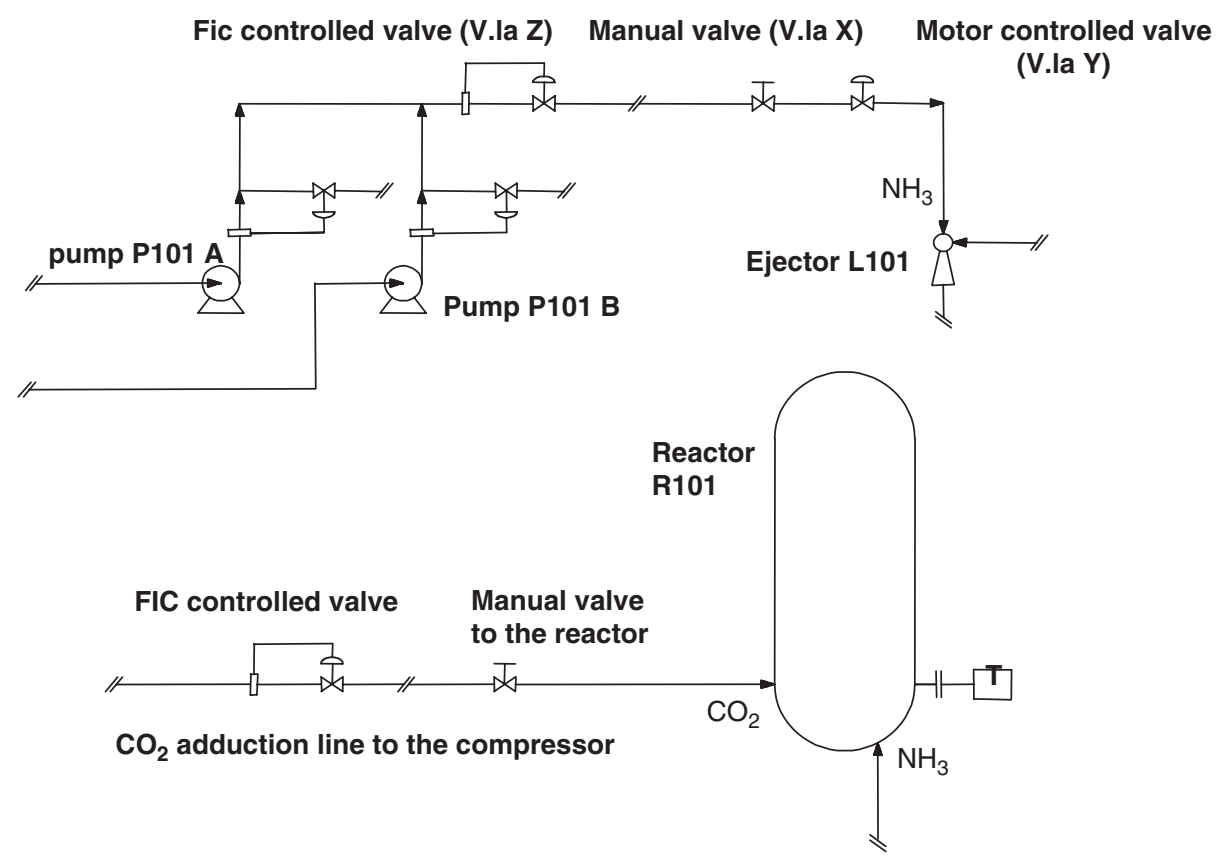

Fig. 7. Sketch of the part of the ammonia urate plant under commissioning.

Table 8

Example of equipment status table

\begin{tabular}{|c|c|c|c|c|c|c|c|c|c|c|}
\hline $\mathrm{CE}$ & Element & State 1 & State 2 & State 3 & State 4 & State 5 & Prob 1 & Prob 2 & Prob 3 & Prob 4 \\
\hline $\mathrm{CE}$ & $\begin{array}{l}\text { FIC } \mathrm{CO}_{2} \text { pre- } \\
\text { existing set }\end{array}$ & Null & Close value & Lower & Higher & & 0 & 0.99999 & 0.000005 & 0.000005 \\
\hline
\end{tabular}

Step 2: open the motor-operated valve on the adduction pipe to the $\mathrm{NH}_{3}$ pump;

Step 3: open the valve on the adduction pipe to the $\mathrm{NH}_{3}$ pump till the expected flow is reached;

Step 4: set the flow value on the FIC and activate the automatic control;

Step 5: open the manual valve on the adduction pipe for the $\mathrm{CO}_{2}$ to the reactor;

Step 6: open the valve on the adduction pipe to the $\mathrm{CO}_{2}$ compressor until the expected flow rate is reached;

Step 7: set the FIC on the expected flow and activate the automatic control.

For each step when the action outcome is an error the correspondent error mode is randomly extracted among those previously selected as available according to the Human Hazop analysis. The action outcome of each step is related to a possible change of the status or position of plant equipments (valve positions, pumps, etc). For this reason two tables have been implemented, one that correlates every possible outcome of the step with the change in the status of the equipment it refers to (Table 8), and one that collect possible pre-existing equipment-status probabilities. For each piece of equipment in fact, several 'action-independent' states are available with the associated probability: the probability of a mechanical failure of a pump is independent from the operator actions, thus the probability of a mechanical failure is a datum provided not by the simulation runs but by other sources (for the case study failure rates provided by the contractor have been used).

\section{Discussion of results}

The results obtained through the simulation campaign for the three selected scenarios are reported in Tables 9 and 10. The maximum value for the probability of failure in task execution refers to the case for which the lower PSFs values has been simulated (worst case scenario) and it corresponds to $20.1 \%$ while the best value (lower probability of failure) is in correspondence with the optimum 
Table 9

Success probability $p$ th obtained through simulation

\begin{tabular}{llll}
\hline & 5 th Percentile & Mean value & 95 th Percentile \\
\hline Worst case & $79.63 \times 10^{-2}$ & $79.88 \times 10^{-2}$ & $80.13 \times 10^{-2}$ \\
Nominal case & $93.12 \times 10^{-2}$ & $93.28 \times 10^{-2}$ & $93.43 \times 10^{-2}$ \\
Optimal case & $94.57 \times 10^{-2}$ & $94.71 \times 10^{-2}$ & $94.84 \times 10^{-2}$ \\
\hline
\end{tabular}

Table 10

Operator error probability $q$ obtained through simulation

\begin{tabular}{lccc}
\hline & 5 th percentile & Mean value & 95th percentile \\
\hline Worst case & $19.87 \times 10^{-2}$ & $20.12 \times 10^{-2}$ & $20.37 \times 10^{-2}$ \\
Nominal case & $6.57 \times 10^{-2}$ & $6.73 \times 10^{-2}$ & $6.88 \times 10^{-2}$ \\
Optimal case & $5.16 \times 10^{-2}$ & $5.30 \times 10^{-2}$ & $5.43 \times 10^{-2}$ \\
\hline
\end{tabular}

configuration of the PSFs (optimal case scenario) and equals $5.3 \%$ (Table 9 ).

Referring to the nominal case, it is possible to analyse the distribution of the HEP associated with the execution of each step of the task (Figs. 8 and 9). The most critical steps are those related to the setting of the flow value on the FIC and the automatic control activation (Step 4 and Step 7).

Furthermore, the simulator allows to consider the HEP for the recovery phase in relation to each step of the procedure, as shown in Fig. 10. The recovery is meant to be applied only to correct erroneous states of the equipment in consequences of operator failures. The equipment that can be object of recovery is the one used or considered in the specific task steps. The results are consistent with the real situation where the recovery fails for $100 \%$ of the actions (Step 1 and Step 4) because the equipment is found to be in

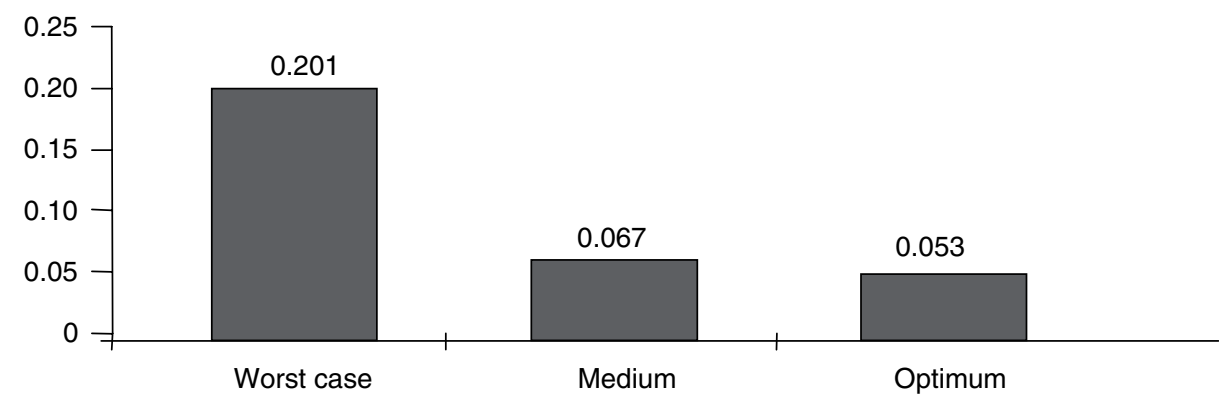

Fig. 8. HEP in completing the procedure for each scenario.

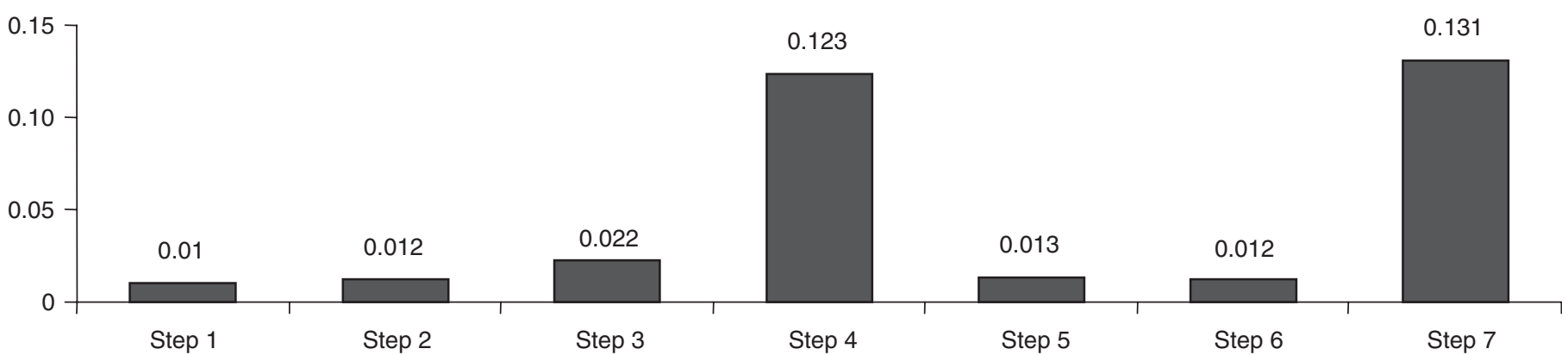

Fig. 9. HEP in completing each step of the procedure for the nominal case scenario.

Error Probability in Recovery phase

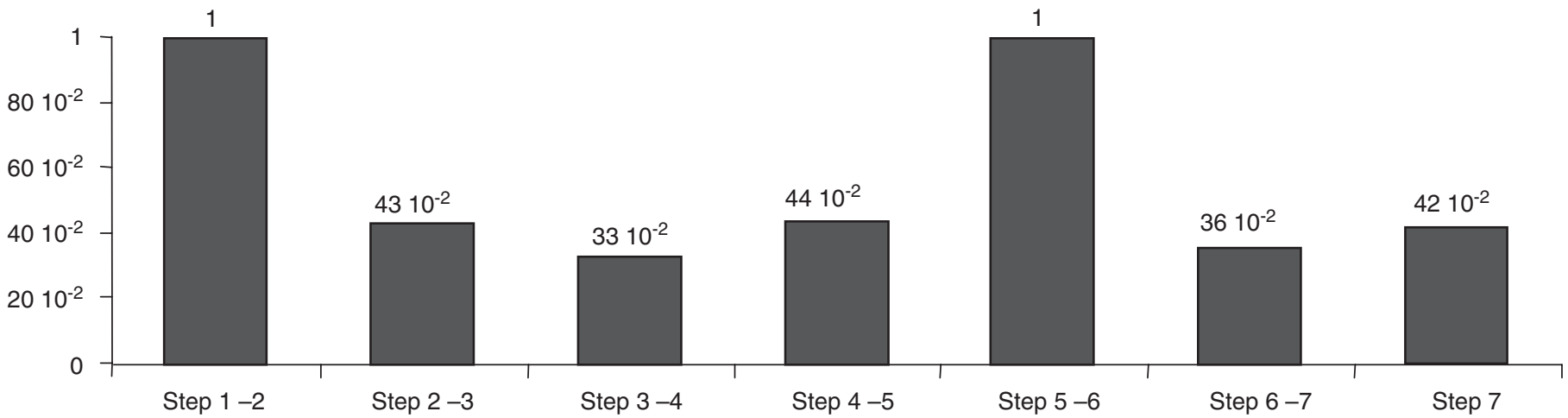

Fig. 10. HEPs for the recovery phase regarding the nominal case scenario. 
Table 11

HEART generic task chosen for comparison, whose nominal probability has been modified according to the error-producing conditions considered for the task

\begin{tabular}{lllll}
\hline Code & Description & Nominal probability & 5th Percentile & 95th Percentile \\
\hline F & $\begin{array}{l}\text { Restore or shift a system to original or new } \\
\text { state following procedures, with some } \\
\text { checking }\end{array}$ & 0.109 & 0.029 & 0.253 \\
\hline
\end{tabular}

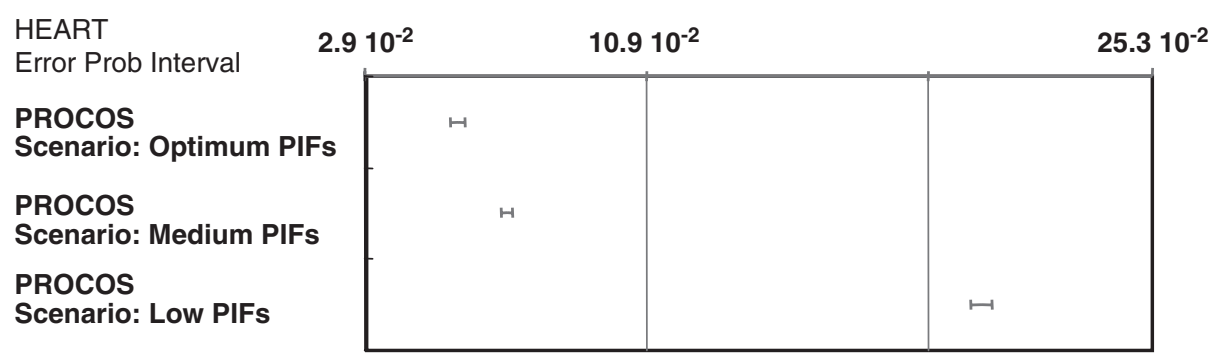

Fig. 11. Comparison of HEPs provided by PROCOS with HEART estimates for the same task.

the wrong position and/or where the manoeuvring of manual valves is not supervised from the control room.

In order to have a first validation of the simulation model it is possible to compare the quality of the quantitative results obtained with the estimates provided by a traditional HRA approach. To this end the human error assessment and reduction technique (HEART) [25] has been chosen, as its quantitative results have been applied to many PSA. In order to compare the results of the simulator the task reported in Table 11 has been chosen among those for which HEART can provide an error probability interval; the nominal value has been then modified according to the error-producing condition proposed by the HEART method in order to adapt the nominal case to the actual case under evaluation.

The HEP interval obtained with the HEART method ranges from 0.029 to 0.25 . This interval has been then compared with the error probability intervals obtained for the three scenarios analysed through the simulation. The upper interval in Fig. 11 is the HEART HEP interval while the smaller ones below are the one obtained for the scenario analysed using PROCOS for the same task. It is apparent that the intervals obtained with the simulator are more narrow than the one provided by HEART and are all contained within the HEART interval, coherently with the scenario they referrer to (the worst case interval is closed to the 95th percentile of the HEART interval, the optimal case interval is closed to the 5th percentile, and the nominal case interval is within the first half of the HEART interval).

\section{Conclusions}

As far as the improvement process is concerned, the simulator makes it possible to directly evaluate how a corrective action influences the probability of success (or failure) of a critical activity. Furthermore, up to now the recovery phase has not been the focus of attentive safety assessment, especially with cognitive-based methodology, even if error handling is stated as one of the most important areas for improving safety performance in complex operations, like commissioning. The simulator is then a new attempt and a contribution in this direction.

The approach is semi-static and therefore it is able to take into consideration different contexts by modifying the PSF involved and the table regarding the equipment and the subtask analysis of the action to be simulated, without requiring a difficult and expensive modelling phase for the plant the operator has to interact with. In addition the output of the simulator can also be directed at quantifying the error types, or the error occurrences as a whole only if the results have to be included in a fault tree; thus enabling the tool to be integrated with the most commonly used risk assessment methodologies.

The simulator in this early stage does not perform a time-dependent simulation process. As a further development the simulation code should be modified so that possible time windows can be considered for both action and recovery execution. Stepping forward in its development some sensitivity analysis has still to be performed on the main elements on which the simulator is based (blocks of the flow chart, decision block criteria, PSF importance) in order to test the robustness of the method.

\section{Acknowledgements}

The authors would like to thank Elisa De Grandis for her precious support, Gianmario Gallarati and Giovanni Corti for the effort they put in contributing to develop the simulator during their degree programme. 


\section{Appendix A.}

Recovery flow chart.

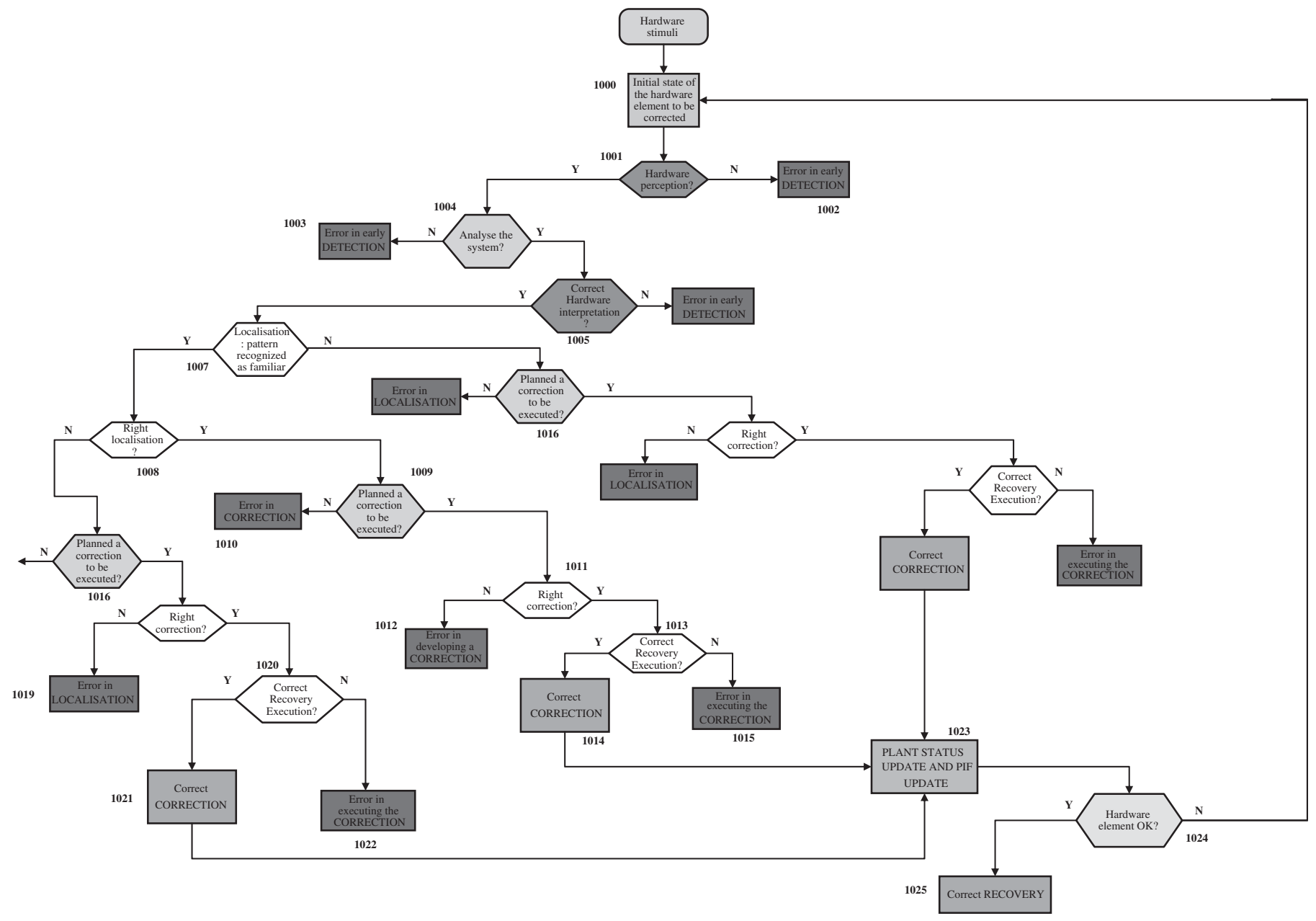

\section{References}

[1] Hollnagel E, Marsden A. Further development of the phenotypegenotype classification scheme for the analysis of human erroneous actions. JRC-European Commission-EUR EN 1996.

[2] Mosleh A, Chang YH. Model-based human reliability analysis: prospects and requirements. Reliab Eng Syst Safety 2004;83: 241-53.

[3] Straeter O. On the way to assess errors of commission. Reliab Eng Syst Safety 2004;83:129-38.

[4] Hollnagel E. Human reliability analysis context and control. London: Academic Press; 1993.

[5] Cacciabue PC. Modelling and simulation of human behaviour in system control. London: Springer \& Verlag; 1998.

[6] Baron S, Zacharias G, Muralidharan R, Lancraft R. PROCRU: a model for analyzing fligth crew procedures in approach to landing. CR-152397, NASA 1980.

[7] Woods DD, Roth EM, People HE. Cognitive environment simulation: an artificial intelligence system for human performance assessment. technical report NUREG-CR-4862, US Regulatory Commission, Washington DC, US, 1987.

[8] Cacciabue PC, Decortis F, Drozdowicz B, Masson M, Nordvik JP. COSIMO: a cognitive simulation model of human decision making and behaviour in accident management of complex plants. IEEE Trans Syst Man Cybern IEEE-SMC 1992;22(5):1058-74.

[9] Reason J. Human error. Cambridge, UK: Cambridge University Press; 1990

[10] Rasmussen J. Information processes and human machine interaction. An approach to cognitive engineering. Amsterdam: Elsevier-North Holland; 1986.

[11] Corker KM, Smith B. An architecture and modelling for cognitive engineering simulation analysis: application to advanced aviation analysis. In: Proceedings of ninth AAIA conference on computing in aerospace, San Diego, CA, 1993.

[12] Sasou K, Takano K, Yoshimura S, Haroko K, Kitamura M. Modelling and simulation of operator team behaviour in nuclear power plants. In: Proceedings of the HCI international '95, Tokyo, 1995.

[13] Mauri C, Owen D, Baranzini D. Model of human machine integrated system. AITRAM Deliverable D04.1 WP4, fifth framework programme, 2001.

[14] Blom HAP, Daams J, Nijhuis HB. Human cognition modelling in ATM safety assessment. In: Proceedings of the third USA/Europe air traffic management R\&D seminar, Napoli, 2000.

[15] Shu Y, Futura K, Kondo S. Team performance modelling for HRA in Dynamic situations. Reliab Eng Syst Safety 2002;78:111-21. 
[16] De Grandis E. Uno Strumento di Simulazione di un Team per Studi Prospettici di Sicurezza in Campo Aeronautico. Pubblicazione speciale No. I.03.64. Ispra: JRC; 2003 [in Italian].

[17] Cagno E, Caron F, Mancini M. Risk analysis in plant commissioning: the Multilevel HAZOP. Reliab Eng Syst Safety 2002;77: $309-23$.

[18] Edwards E. Human factors in aviation. London: Academic Press; 1988.

[19] Kontogiannis T. A framework for analysis of cognitive reliability in complex systems: a recovery centred approach. Reliab Eng Syst Safety 1997;58:233-48.

[20] Hawkins FH. Human factors in flight. Aldershot, UK: Gower Technical Press; 1987.

[21] Wickens CD. Engineering psychology and human performance. New York: Harper Collins Publishers; 1992.

[22] Embrey DE, Humphreys PC, Rosa EA, Kirwan B, Rea K. SLIMMAUD: an Approach to assessing human error probabilities using structured expert judgement. NUREG/CR-3518. Washington, US: USNRC; 1984.

[23] Fujita Y, Hollnagel E. Failures without errors: quantification of context in HRA. Reliab Eng Syst Safety 2004;83:141-51.

[24] Swain AD, Guttmann HE. Handbook on human reliability analysis with emphasis on nuclear power plant application. NUREG/CR1278, SAND 08-0200 R X, AN, 1983.
[25] Williams JC. HEART - a proposed method for assessing and reducing human error. In: Proceedings of the ninth advances in reliability technology symposium, University of Bradford, 1986.

Paolo Trucco, Ph.D. is Associate Professor of Ergonomics and Safety Engineering at Politecnico di Milano. His main research interests are Quantitative Risk Assessment, Human and Organisational Factors Analysis, vulnerability of complex systems. He is responsible for a number of research projects in the Safety Engineering and Human Factors area. He is the author of more than 100 publications including books and papers. His previous experience includes consultancy in the field of industrial safety and ergonomics for both large companies and SMEs in the process industry, transportation and energy sectors.

Maria Chiara Leva is a Ph.D. student of the Politecnico di Milano under the programme Planning and management of human factors in transport systems safety funded by D'Appolonia Spa. Her previous experience started in the International Institute for Applied System Analysis (IIASA) in Laxenburg, Austria while studying Human Error Analysis in Industrial Accidents and Safety Management Systems, in relation to the EU Seveso II Directive. She is also involved in research projects concerning Human Reliability Analysis in the Nuclear and the Maritime domains. 